\title{
Call for crop improvement Analysis articles
}

\begin{abstract}
We offer to publish Analyses of genomic and phenotypic data that present new concepts and strategies for the improvement of crop plant yield and nutritional value via the introduction, selection and use of genetic variation within the context of changes in markets, climate, water use and agronomic practices.
\end{abstract}

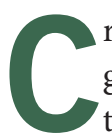
rop improvement through genetics and breeding has had great success from a small number of strategies: selection of inbred lines, cross-breeding to maximize desirable heterozygous combinations and introduction of short-stem mutations to produce robustly growing cereals that maximize fertilizer use. Research into phenotypic traits conferred by mutations, quantitative trait-associated variants and genotypes selected via genetic markers has played a part in yield improvement, but we think that there is much more to gain from the recent flood of genomic research that will only come from new bioinformatic models and genetic strategies, as well as from crossover when approaches in genomic plant breeding for one crop are applied to another.

To start discussion (but not to restrict the creativity of authors in coming up with additional approaches), we propose the generation and testing of three classes of model as being of importance to the future of genetic approaches to plant yield and nutrition. First, modeling plant developmental architecture is a key way to integrate genetic contributions to yield. Second, we need metabolic and physiological models of the ways variation in the biochemical pathways responsible for protein, carbohydrate, lipid and micronutrient production interact to impinge on yield. Third, we need more understanding of the ways genotypic architecture itself responds to variation, starting with a mechanistic genomic understanding of phenomena such as epistasis, heterosis, expression dominance and trait stability. The effects of diseases seem to be a fourth class for analysis, but perturbation of the system by other environmental stresses might usefully be built into all of the models.

There is considerable concern arising from predictions that rates of gain in crop yield will be cut by short-term changes in environmental conditions (Environ. Res. Lett. 9, 074003, 2014), particularly in the context of continuing increase in the human population (Nat. Clim. Change 4, 287-291, 2014; for current statistics, see http://faostat3.fao.org/home/).
Some useful suggestions to avert this predicted crisis have been made, including diverting basic research effort to model plants where results can more readily be translated to cereal crop breeding programs (Nat. Plants 1, 15062, 2015). Another promising strategy is to target research and development in agriculture to the geographical regions that have the most potential for growth and human benefit (Nat. Plants 1, 14014, 2015). In sub-Saharan Africa, one such region of enormous growth potential, genetics and breeding strategies must address not only yield at harvest but also disease resistance, storage and transport traits, existing and potential agronomic practices, infrastructural changes and the overall economic balance of locally consumed and export crops. To be useful for genetics in this context, models need interdisciplinary input and scrutiny, issues we will take seriously in our peer review strategy for such publications.

In numerous Editorials, we have endorsed a strategy of public data deposition and imaginative data reanalysis to boost scientific productivity in both biomedical and agricultural genomics with increased rigor and transparency (most recently, Nat. Genet. 47, 99, 2015).

The response from the agricultural genomics community this year has been particularly encouraging, in that the majority of authors queried have responded positively to requests to publish a Data Descriptor (http://www.nature.com/sdata/). This persuades us to provide an incentive for the mining and analysis of these data sets and a venue for two types of publication in this field, namely Analysis articles for new ideas from data reuse and Perspectives presenting new priorities from literature review. We will of course consider proposals and submissions of Analyses and Perspectives at any time, but, to maximize the impact of this important area by coordinating publication, we ask that the first round of presubmission inquiries via our online database (http://mts-ng.nature.com/cgi-bin/main.plex) be submitted by 1 October for submission this year. 